Journal of

\title{
Mechanics of
}

Materials and Structures

ELASTIC INDENTATION PROBLEMS IN THIN FILMS ON SUBSTRATE SYSTEMS

Roberta Sburlati

Volume 1, № 3

March 2006

mathematical sciences publishers 


\title{
ELASTIC INDENTATION PROBLEMS IN THIN FILMS ON SUBSTRATE SYSTEMS
}

\author{
ROBERTA SBURLATI
}

\begin{abstract}
In this paper an analytical solution of an elastic isotropic thin-film on an elastic substrate under an axisymmetric loading on the plane surface is presented. The analysis is intended to model the micronanoindentation tests to evaluate some of the relevant properties of thin films and provide information about the influence of interface conditions between the film and the substrate.

The theoretical solution of the equations of three-dimensional elasticity is obtained by using Dini and Fourier-Bessel expansions for the displacement field. To describe the elastic mechanical interaction between the indenter and the film for low load, we make use of the pressure distribution for contact between two homogeneous bodies, and the corresponding displacement field is solved in explicit form. The contact law is obtained with two different ideal interface conditions between the film and the substrate: perfectly bonded and frictionless contact.
\end{abstract}

This form of the elastic solution may be utilized for different axisymmetric pressure distributions performed to model the interaction between the indenter and the film, thus obtaining an analytical framework for comparing experimental and numerical results.

\section{Introduction}

The growing importance of nanomicroscale materials has recently rekindled the interest in thin-film technology and the mechanical properties of a thin solid film deposited on a substrate. The interaction between a rigid indenter and an elastic half-space is a classical problem of contact mechanics that was studied by Sneddon [1966] by using the integral transforms technique. When a solid thin film is deposited over the substrate, the problem becomes more complex and the elastic response of the film subjected to indentation can be categorized according to the film/substrate material properties. In the first case, the film is soft in comparison with the substrate stiffness (soft-film); in the second case, the elastic properties of the film and the substrate are comparable; and in the third case the thin film is stiffer (hard film). For the first case, some investigators assume a mathematical artifice that simplifies the analysis: the substrate is approximated as rigid [Matthewson

Keywords: elasticity, contact mechanics, thin film, nanoindentation. 
1981]. However, for the other cases, a correct evaluation of the mechanical behavior of the indentation problem requires knowledge of the effects of elastic properties of both the film and the substrate.

Experimental indentation tests are frequently used to measure the elastic properties of materials and to investigate the role of adhesion forces. In order to experimentally measure "film-only" properties, a commonly used rule of thumb for the substrate influences is to limit the indentation depth to less than $10 \%$ of the film thickness. Actually, some studies have shown that the critical parameter to take into account in the experimental indentation tests is the ratio between the thickness of the film and the contact area radius $a$; also in this case, it is proposed that the limit is less than $10 \%$ in order to use the monolithic theory. While using this rule is experimentally feasible for films that are greater than about a micrometer in thickness, this approach cannot be used for very thin films. Hence these assumptions, frequently accepted in the experimental tests for a large class of materials, are not applicable at the nanoscale [VanLandingham 2003; Fischer-Cripps 2004].

Intense studies have taken into account the adhesion forces which arise between the indenter and the film and on the interface film/substrate, and which give rise to an increasing of the contact area with respect to the contact without adhesion case [Maugis 1999].

In this work, we consider an isotropic film coating an isotropic elastic substrate subjected to an axisymmetric loading condition which simulates the presence of an indenter of assigned form in a quasi-static indentation test. We make two main assumptions. First, in view of the local character of the indentation problem, we introduce a suitable parameter $b$ representing the radius in which the contact phenomenon is contained. In such a way, we write the components of the displacement field by means of Dini and Fourier-Bessel expansions, so avoiding the use of the Hankel integral transforms and, consequently, the problem of their inversions [Yu et al. 1990]. We shall subsequently show that, under suitable conditions, the specific value assumed for the parameter $b$ is not relevant. Second, since the original mixed boundary value indentation problem leads to dual integral equations that, due to their complexity, can only be solved numerically by means of the Fredholm integral technique, we have changed the boundary conditions by assuming a preassigned distribution of traction on the free surface; this assumption is also introduced in the paper of Li and Chou [1997] within the framework of the Hankel integral transform technique.

In so doing, we solve the elastic problem of a thin film coating/substrate system under a prescribed axisymmetric load by using a Dini and a Fourier-Bessel expansion for the radial and the vertical component of the displacement field respectively. The elastic response of the film is analyzed with two different interface conditions between the substrate and the film to bound the real case: frictionless contact 
and perfectly bonded. We also analyze the case in which the elastic properties of the film are very different from the substrate ones (soft-film/hard substrate). We get an explicit analytical form for the displacement and stress fields in terms of Fourier-Bessel expansions depending on some coefficients related to the form of the indenter and to the preassigned pressure distribution suitable to model the interaction film-indenter.

Although we do not assume specific restrictions on the thickness of the film, we consider the film "thin" for two reasons: first, because of its experimental relevance in micro and nanoindentation tests; and second, because we are interested in evaluating substrate effects that are not relevant for thick films (see the classical half-space theory [Sneddon 1966]).

Finally, we remark that the indentation test involves plastic deformation; indeed if we consider the loading of an initially flat specimen with a spherical indenter, after an initial elastic response at low loads, there is an elastic-plastic deformation. However, the elastic three-dimensional solution presented in this paper may be used to describe the low load elastic film response when the classical half-space solution is no longer true due to the presence of the film thickness.

The elastic solution may also be used to describe the unloading curve by adopting the Oliver and Pharr method; of course, one has to take into account the residual depth at complete unload [Oliver and Pharr 1992; Field and Swain 1993].

\section{Problem formulation}

In this section we study the indentation problem of a rigid, frictionless axisymmetric indenter on a circular thin-film/substrate system of radius $b$. The film is assumed to be either in frictionless contact or perfectly bonded to an elastic substrate. The thickness of the finite film overlaying the substrate is denoted as $h$. We choose a cylindrical coordinate system $(0, r, \vartheta, z)$ such that $z$ is parallel to the generator of the indenter and the origin 0 is placed at the first contact point between the indenter and the film.

Due to the local character of the effect, we limit our study to the volume of a cylinder of radius $b$ (the centre is the origin); by assuming that $b$ is sufficiently large with respect to the radius $a$ of the contact area, we can suppose that, for $r \geq b$, the vertical displacement does not change in the presence of the indenter; in other words, we assume $w(b, z)=0$.

By considering an axisymmetric loading condition on the plane surface, we limit our attention to the radial and the transversal displacement fields $u^{(i)}=u^{(i)}(r, z)$ and $w^{(i)}=w^{(i)}(r, z)$ for the film and the substrate respectively (with $i=f, s$ ); by using a Dini expansion in $r$ for $u^{(i)}(r, z)$ and a Fourier-Bessel expansion in $r$ for $w^{(i)}(r, z)$ (see [Watson 1944], p. 576-577, equation (5) with $v=1, H=1$ in 
equation (1), respectively), we write

$$
\begin{aligned}
& u^{(f)}(r, z)=-\sum_{j=1}^{\infty} g_{j}^{(f)}(z) \phi_{j} J_{1}\left(\phi_{j} r\right), \quad w^{(f)}(r, z)=\sum_{j=1}^{\infty} f_{j}^{(f)}(z) J_{0}\left(\phi_{j} r\right), \\
& u^{(s)}(r, z)=-\sum_{j=1}^{\infty} g_{j}^{(s)}(z) \phi_{j} J_{1}\left(\phi_{j} r\right), \quad w^{(s)}(r, z)=\sum_{j=1}^{\infty} f_{j}^{(s)}(z) J_{0}\left(\phi_{j} r\right) .
\end{aligned}
$$

The functions $g_{j}^{(s)}(z)$ and $f_{j}^{(s)}(z)$ denote the variations of the displacements through the thickness of the film and of the substrate; we have put

$$
\phi_{j}=\frac{Z_{j}^{(0)}}{b}
$$

where $Z_{j}^{(0)}$ denote, for $j=1,2,3, \ldots$, the positive zeros of the zero-order Bessel function $J_{0}(r)$.

In this paper we assume that the film and the substrate are both isotropic; the linear elasticity equations therefore assume the form

$$
\begin{array}{r}
\left(\lambda_{i}+2 \mu_{i}\right)\left(\frac{\partial^{2}}{\partial r^{2}} u^{(i)}+\frac{1}{r} \frac{\partial}{\partial r} u^{(i)}-\frac{1}{r^{2}} u^{(i)}+\frac{\partial^{2}}{\partial z \partial r} w^{(i)}\right)+\mu_{i}\left(\frac{\partial^{2}}{\partial z^{2}} u^{(i)}-\frac{\partial^{2}}{\partial z \partial r} w^{(i)}\right) \\
=0 \\
\left(\lambda_{i}+2 \mu_{i}\right)\left(\frac{\partial^{2}}{\partial z^{2}} w^{(i)}+\frac{1}{r} \frac{\partial}{\partial z} u^{(i)}+\frac{\partial^{2}}{\partial z \partial r} u^{(i)}\right)+\mu_{i}\left(\frac{\partial^{2}}{\partial r^{2}} w^{(i)}-\frac{\partial^{2}}{\partial z \partial r} u^{(i)}\right) \\
-\mu_{i} \frac{1}{r}\left(\frac{\partial}{\partial z} u^{(i)}-\frac{\partial}{\partial r} w^{(i)}\right)=0,
\end{array}
$$

where $\lambda_{i}$ and $\mu_{i}$ are the Lamé moduli.

By substituting the displacement field (1), (2) into these equations, we get the differential equations

$$
\begin{aligned}
&\left(\lambda_{i}+2 \mu_{i}\right) \frac{d^{2}}{d z^{2}} f_{j}^{(i)}+\phi_{j}^{2}\left(\left(\lambda_{i}+\mu_{i}\right) \frac{d}{d z} g_{j}^{(i)}-\mu_{i} f_{j}^{(i)}\right)=0, \\
& \mu_{i} \frac{d^{2}}{d z^{2}} g_{j}^{(i)}-\left(\lambda_{i}+\mu_{i}\right) \frac{d}{d z} f_{j}^{(i)}-\phi_{j}^{2}\left(2 \mu_{i}+\lambda_{i}\right) g_{j}^{(i)}=0,
\end{aligned}
$$

whose solution has the form

$$
\begin{aligned}
& f_{j}^{(f)}(z)=C_{1}^{(j)} \cosh \left(\phi_{j} z\right)+C_{2}^{(j)} \sinh \left(\phi_{j} z\right)+C_{3}^{(j)} z \cosh \left(\phi_{j} z\right)+C_{4}^{(j)} z \sinh \left(\phi_{j} z\right), \\
& g_{j}^{(f)}(z)=-\frac{1}{\phi_{j}} C_{1}^{(j)} \sinh \left(\phi_{j} z\right)-\frac{1}{\phi_{j}} C_{2}^{(j)} \cosh \left(\phi_{j} z\right)
\end{aligned}
$$




$$
\begin{aligned}
& -\frac{1}{\phi_{j}} C_{3}^{(j)}\left(\frac{1}{\phi_{j}} \frac{\lambda_{f}+3 \mu_{f}}{\lambda_{f}+\mu_{f}} \cosh \left(\phi_{j} z\right)+z \sinh \left(\phi_{j} z\right)\right) \\
& -\frac{1}{\phi_{j}} C_{4}^{(j)}\left(\frac{1}{\phi_{j}} \frac{\lambda_{f}+3 \mu_{f}}{\lambda_{f}+\mu_{f}} \sinh \left(\phi_{j} z\right)+z \cosh \left(\phi_{j} z\right)\right),
\end{aligned}
$$

$f_{j}^{(s)}(z)=Q_{1}^{(j)} \cosh \left(\phi_{j} z\right)+Q_{2}^{(j)} \sinh \left(\phi_{j} z\right)+Q_{3}^{(j)} z \cosh \left(\phi_{j} z\right)+Q_{4}^{(j)} z \sinh \left(\phi_{j} z\right)$, $g_{j}^{(s)}(z)=-\frac{1}{\phi_{j}} Q_{1}^{(j)} \sinh \left(\phi_{j} z\right)-\frac{1}{\phi_{j}} Q_{2}^{(j)} \cosh \left(\phi_{j} z\right)$

$$
\begin{aligned}
& -\frac{1}{\phi_{j}} Q_{3}^{(j)}\left(\frac{1}{\phi_{j}} \frac{\lambda_{s}+3 \mu_{s}}{\lambda_{s}+\mu_{s}} \cosh \left(\phi_{j} z\right)+z \sinh \left(\phi_{j} z\right)\right) \\
& -\frac{1}{\phi_{j}} Q_{4}^{(j)}\left(\frac{1}{\phi_{j}} \frac{\lambda_{s}+3 \mu_{s}}{\lambda_{s}+\mu_{s}} \sinh \left(\phi_{j} z\right)+z \cosh \left(\phi_{j} z\right)\right) .
\end{aligned}
$$

in the film and in the substrate, respectively. The coefficients $C_{1}^{(j)}, C_{2}^{(j)}, C_{3}^{(j)}, C_{4}^{(j)}$ and $Q_{1}^{(j)}, Q_{2}^{(j)}, Q_{3}^{(j)}, Q_{4}^{(j)}$ will be uniquely determined by imposing the boundary and interface conditions that will be introduced in the next section.

The displacement field is obtained by considering the sum over all values of $j$; thus

$$
\begin{aligned}
u^{(f)}(r, z)=\sum_{j=1}^{\infty}( & C_{1}^{(j)} \sinh \left(\phi_{j} z\right)+C_{2}^{(j)} \cosh \left(\phi_{j} z\right) \\
& +C_{3}^{(j)}\left(\frac{1}{\phi_{j}} \frac{\lambda_{f}+3 \mu_{f}}{\lambda_{f}+\mu_{f}} \cosh \left(\phi_{j} z\right)+z \sinh \left(\phi_{j} z\right)\right) \\
& \left.+C_{4}^{(j)}\left(\frac{1}{\phi_{j}} \frac{\lambda_{f}+3 \mu_{f}}{\lambda_{f}+\mu_{f}} \sinh \left(\phi_{j} z\right)+z \cosh \left(\phi_{j} z\right)\right)\right) J_{1}\left(\phi_{j} r\right)
\end{aligned}
$$

$$
\begin{aligned}
w^{(f)}(r, z)=\sum_{j=1}^{\infty}\left(C_{1}^{(j)} \cosh \left(\phi_{j} z\right)+C_{2}^{(j)} \sinh \left(\phi_{j} z\right)\right. & \\
& \left.+C_{3}^{(j)} z \cosh \left(\phi_{j} z\right)+C_{4}^{(j)} z \sinh \left(\phi_{j} z\right)\right) J_{0}\left(\phi_{j} r\right) .
\end{aligned}
$$

$$
\begin{aligned}
u^{(s)}(r, z)=\sum_{j=1}^{\infty}( & Q_{1}^{(j)} \sinh \left(\phi_{j} z\right)+Q_{2}^{(j)} \cosh \left(\phi_{j} z\right) \\
& +Q_{3}^{(j)}\left(\frac{1}{\phi_{j}} \frac{\lambda_{s}+3 \mu_{s}}{\lambda_{s}+\mu_{s}} \cosh \left(\phi_{j} z\right)+z \sinh \left(\phi_{j} z\right)\right) \\
& \left.+Q_{4}^{(j)}\left(\frac{1}{\phi_{j}} \frac{\lambda_{s}+3 \mu_{s}}{\lambda_{s}+\mu_{s}} \sinh \left(\phi_{j} z\right)+z \cosh \left(\phi_{j} z\right)\right)\right) J_{1}\left(\phi_{j} r\right),
\end{aligned}
$$




$$
\begin{aligned}
w^{(s)}(r, z)=\sum_{j=1}^{\infty}\left(Q_{1}^{(j)} \cosh \left(\phi_{j} z\right)\right. & +Q_{2}^{(j)} \sinh \left(\phi_{j} z\right) \\
& \left.+Q_{3}^{(j)} z \cosh \left(\phi_{j} z\right)+Q_{4}^{(j)} z \sinh \left(\phi_{j} z\right)\right) J_{0}\left(\phi_{j} r\right) .
\end{aligned}
$$

\section{Boundary and interface conditions}

In order to explicitly find the form of the elastic solution (5)-(8), we investigate the boundary condition on the free surface of the film and on the interface film/substrate.

Due to the complexity of the indentation problem, an axisymmetric normal pressure and vanishing shear stress components are assumed on the free surface, in $z=0$, as follows

$$
\sigma_{z z}^{(f)}(r, 0)=p(r) \quad \text { and } \quad \sigma_{r z}^{(f)}(r, 0)=0 .
$$

It is convenient to write the normal pressure distribution $p(r)$ with a FourierBessel expansion in the form

$$
p(r)=\sum_{j=1}^{\infty} A_{j} J_{0}\left(\phi_{j} r\right), \quad \text { where } \quad A_{j}=\frac{2 \int_{0}^{a} r p(r) J_{0}\left(\phi_{j} r\right) d r}{b^{2} J_{1}\left(b \phi_{j}\right)^{2}} .
$$

A detailed discussion on the expansion convergence can be found in [Watson 1944]. On the other hand, in the substrate, for large $z$, we assume

$$
\lim _{z \rightarrow \infty} u^{(s)}(r, z)=0 \quad \text { and } \quad \lim _{z \rightarrow \infty} w^{(s)}(r, z)=0 .
$$

These conditions give the following equations: $Q_{1}^{(j)}=-Q_{2}^{(j)}$ and $Q_{3}^{(j)}=-Q_{4}^{(j)}$.

Now we introduce two different conditions in the interface zone; the film is assumed to be in frictionless contact or perfectly bonded to a substrate. The solutions obtained for both these ideal cases are introduced to bound the real cases where the contact between the film and the substrate is neither frictionless nor perfectly bonded.

3.1. Perfectly bonded case. On the interface surface $z=h$, we require the continuity of displacement and traction components as follow

$$
\begin{array}{ll}
{[w(r, h)]=0,} & {[u(r, h)]=0,} \\
{\left[\sigma_{z z}(r, h)\right]=0,} & {\left[\sigma_{r z}(r, h)\right]=0 .}
\end{array}
$$

The conditions in (12), (13) together with (9) allow us to get the explicit form of the remaining unknown coefficients $C_{\alpha}^{(j)}, \alpha=1, \ldots, 4$ and $Q_{\alpha}^{(j)}, \alpha=2$, in terms of the $A_{j}$ coefficients. 
In the case of a rigid substrate, by putting $\lambda^{(f)}=\lambda$ and $\mu^{(f)}=\mu$, the interface conditions

$$
w(r, h)=0 \quad \text { and } \quad u(r, h)=0
$$

give rise to the following simplified forms for the coefficients:

$$
\begin{aligned}
& C_{1}^{(j)}=-\frac{(\lambda+2 \mu)\left((\lambda+3 \mu) \sinh \left(2 \phi_{j} h\right)-2 \phi_{j} h(\lambda+\mu)\right)}{4 \mu \phi_{j}\left((\lambda+\mu)(\lambda+3 \mu) \sinh ^{2}\left(\phi_{j} h\right)+\phi_{j}^{2} h^{2}(\lambda+\mu)^{2}+(2 \mu+\lambda)^{2}\right)} A_{j}, \\
& C_{2}^{(j)}=\frac{(\lambda+2 \mu)(\lambda+3 \mu) \cosh \left(2 \phi_{j} h\right)+2 \phi_{j}^{2} h^{2}(\lambda+\mu)^{2}+(\lambda+2 \mu)(\lambda+3 \mu)}{4 \mu \phi_{j}\left((\lambda+\mu)(\lambda+3 \mu) \sinh ^{2}\left(\phi_{j} h\right)+\phi_{j}^{2} h^{2}(\lambda+\mu)^{2}+(2 \mu+\lambda)^{2}\right)} A_{j}, \\
& C_{3}^{(j)}=-\frac{(\lambda+\mu)\left((\lambda+3 \mu) \cosh \left(2 \phi_{j} h\right)+(\lambda+\mu)\right)}{4 \mu\left((\lambda+\mu)(\lambda+3 \mu) \sinh ^{2}\left(\phi_{j} h\right)+\phi_{j}^{2} h^{2}(\lambda+\mu)^{2}+(2 \mu+\lambda)^{2}\right)} A_{j}, \\
& C_{4}^{(j)}=\frac{(\lambda+\mu)\left((\lambda+3 \mu) \sinh \left(2 \phi_{j} h\right)-2 h \phi_{j}(\lambda+\mu)^{2}\right)}{4 \mu\left((\lambda+\mu)(\lambda+3 \mu) \sinh ^{2}\left(\phi_{j} h\right)+\phi_{j}^{2} h^{2}(\lambda+\mu)^{2}+(2 \mu+\lambda)^{2}\right)} A_{j} .
\end{aligned}
$$

The coefficients $A_{j}$ can be found by using the expression in (10) for the pressure on the free film surface.

3.2. Frictionless contact. On the interface surface $z=h$, we require the continuity of transversal displacement components, traction components, and null shear traction, as follows:

$$
\begin{array}{rlrl}
{[w(r, h)]} & =0, & \sigma_{r z}^{(i)}(r, h) & =0, \\
{\left[\sigma_{z z}(r, h)\right]} & =0, \quad\left[\sigma_{r z}(r, h)\right]=0 .
\end{array}
$$

Together with (9), these conditions allow us to get the coefficients $C_{\alpha}^{(j)}, \alpha=$ $1, \ldots, 4$ and $Q_{\alpha}^{(j)}, \alpha=2,3$, in terms of the $A_{j}$.

For a rigid substrate the coefficient expressions assume the simplified form

$$
\begin{aligned}
& C_{1}^{(j)}=-\frac{(\lambda+2 \mu)\left(\cosh \left(2 \phi_{j} h\right)-1\right)}{2 \mu \phi_{j}(\lambda+\mu)\left(\sinh \left(2 \phi_{j} h\right)+2 \phi_{j} h\right)} A_{j}, \\
& C_{2}^{(j)}=\frac{(\lambda+2 \mu) \sinh \left(2 \phi_{j} h\right)+(\lambda+\mu) 2 \phi_{j} h}{2 \mu \phi_{j}(\lambda+\mu)\left(\sinh \left(2 \phi_{j} h\right)+2 \phi_{j} h\right)} A_{j}, \\
& C_{3}^{(j)}=-\frac{\sinh \left(2 \phi_{j} h\right)}{2 \mu\left(\sinh \left(2 \phi_{j} h\right)+2 \phi_{j} h\right)} A_{j}, \\
& C_{4}^{(j)}=\frac{\cosh \left(2 \phi_{j} h\right)-1}{2 \mu\left(\sinh \left(2 \phi_{j} h\right)+2 \phi_{j} h\right)} A_{j} .
\end{aligned}
$$


The displacement field is obtained in explicit form from Equations (5)-(8), by taking into account the coefficients expression $A_{j}$ for detailed preassigned pressure form (10) and the coefficients $C_{\alpha}^{(j)}, \alpha=1, \ldots, 4$ and $Q_{\alpha}^{(j)}, \alpha=2$, for different interface conditions (Sections 3.1, 3.2).

Now we specify the form of the indenter since it is related to the pressure field on the free surface. Specifically, in the following we assume that the pressure distribution is given by the classical solutions for the indentation on an elastic halfspace.

We remark that it is also possible to introduce different pressure distributions to simulate different effects between the indenter and the film (adhesion forces, approximate pressure distribution taking into account the thickness of the films; see [Yang 2003; Chadwick 2002]).

3.2.1. Rigid flat-ended cylindrical punch. We consider the case in which the film is deformed by a flat-ended rigid cylinder with radius $a$. In this case the pressure distribution for the homogeneous half-space is:

$$
p(r)=-p_{0}\left(1-\frac{r^{2}}{a^{2}}\right)^{-\frac{1}{2}}, \quad p_{0}=\frac{P}{2 \pi a^{2}},
$$

where $P$ is the resultant applied load. By using equation (10) the coefficients $A_{j}$ assume the following form

$$
A_{j}=\frac{2 a p_{0} \sin \left(a \phi_{j}\right)}{b^{2} \phi_{j} J_{1}\left(b \phi_{j}\right)^{2}} .
$$

3.2.2. Rigid spherical punch with shallow indentation. We consider a spherical punch by assuming that the radius $a$ of the contact area is much smaller than the radius $R$ of the sphere, that is, $a \ll R$. The pressure distribution, for $r<a$, has the form

$$
p(r)=-p_{0}\left(1-\frac{r^{2}}{a^{2}}\right)^{\frac{1}{2}} \quad \text { with } \quad p_{0}=\frac{3 P}{2 \pi a^{2}} \quad \text { and } \quad a^{3}=\frac{3 P R\left(1-v^{2}\right)}{4 E} .
$$

In this case the coefficients $A_{j}$ are

$$
A_{j}=\frac{2 p_{0}\left(\sin \left(a \phi_{j}\right)-a \phi_{j} \cos \left(a \phi_{j}\right)\right)}{a b^{2} \phi_{j}^{3} J_{1}\left(b \phi_{j}\right)^{2}} .
$$

3.2.3. Rigid conical punch. We consider a right circular cone with semi-vertical angle $\alpha$ whose axis coincides with the $z$-axis and the vertex points downward into the interior of the layer. The pressure distribution under the punch is

$$
p(r)=-p_{0} \cosh ^{-1}\left(\frac{a}{r}\right) \text { with } p_{0}=\frac{P}{\pi a^{2}}, \quad a^{2}=\frac{2 P\left(1-v^{2}\right)}{\pi E \tan \beta} \text { with } \beta=\frac{\pi}{2}-\alpha .
$$


The $j$-th coefficient has the form

$$
A_{j}=\frac{p_{0} a^{2}\left(1-\frac{1}{12} a^{2} \phi_{j}^{2}+\frac{1}{360} a^{4} \phi_{j}^{4}-\cdots\right)}{b^{2} J_{1}\left(b \phi_{j}\right)^{2}} .
$$

This expression was obtained by using an expansion of the $J_{0}(r)$ Bessel function.

\section{Contact law and stress distribution in thin film}

The contact law is obtained by using the explicit form of the transversal displacement (6) in $z=0$ and $r=0$ and can be written in the following compact form

$$
\delta_{\text {film }}=\sum_{j=1}^{\infty} K_{j} A_{j}
$$

Now, by using the explicit form of the coefficients $C_{\alpha}^{(j)}, \alpha=1, \ldots, 4$ and $Q_{\alpha}^{(j)}, \alpha=2$, we get the terms $K_{j}$ in which we take into account the interface conditions; the pressure distribution form allows us to determine the terms $A_{j}$.

In the case of a thin film on a rigid substrate, the terms $K_{j}$ are

$$
\begin{aligned}
K_{j}^{\text {bonded }} & =\frac{\left(v^{2}-1\right)\left((4 v-3) \sinh \left(2 \phi_{j} h\right)+2 \phi_{j} h\right)}{E \phi_{j}\left((4 v-3) \cosh \left(\phi_{j} h\right)^{2}-(2 v-1)^{2}-\phi_{j}^{2} h^{2}\right)}, \\
K_{j}^{\text {contact }} & =\frac{2\left(v^{2}-1\right)\left(\cosh \left(2 \phi_{j} h\right)-1\right)}{E \phi_{j}\left(\sinh \left(2 \phi_{j} h\right)+2 \phi_{j} h\right)},
\end{aligned}
$$

where $v$ is the Poisson's ratio and $E$ the Young's modulus.

The knowledge of the stress field is relevant for the understanding of the failure mechanism of the coating/substrate system. The $j$-th term of the stress components for the case of a rigid substrate can be written as follows:

$$
\begin{aligned}
\sigma_{z z}^{(j)}=2 \mu J_{0}\left(\phi_{j} r\right)\left(C_{1}^{(j)} \phi_{j} \sinh \left(\phi_{j} z\right)\right. & +C_{2}^{(j)} \phi_{j} \cosh \left(\phi_{j} z\right) \\
+C_{3}^{(j)}\left(\frac{\mu}{\lambda+\mu} \cosh \left(\phi_{j} z\right)+\phi_{j} z \sinh \left(\phi_{j} z\right)\right) & \\
& \left.\quad+C_{4}^{(j)}\left(\frac{\mu}{\lambda+\mu} \sinh \left(\phi_{j} z\right)+\phi_{j} z \cosh \left(\phi_{j} z\right)\right)\right),
\end{aligned}
$$




$$
\begin{gathered}
\sigma_{r z}^{(j)}=2 \mu J_{1}\left(\phi_{j} r\right)\left(C_{1}^{(j)} \phi_{j} \cosh \left(\phi_{j} z\right)+C_{2}^{(j)} \phi_{j} \sinh \left(\phi_{j} z\right)\right. \\
+C_{3}^{(j)}\left(\frac{\lambda+2 \mu}{\lambda+\mu} \sinh \left(\phi_{j} z\right)+\phi_{j} z \cosh \left(\phi_{j} z\right)\right) \\
\left.+C_{4}^{(j)}\left(\frac{\lambda+2 \mu}{\lambda+\mu} \cosh \left(\phi_{j} z\right)+\phi_{j} z \cosh \left(\phi_{j} z\right)\right)\right), \\
\sigma_{r r}^{(j)}=\frac{2 \mu}{r}\left(C_{1}^{(j)} \sinh \left(\phi_{j} z\right)+C_{2}^{(j)} \cosh \left(\phi_{j} z\right)\right)\left(-\phi_{j} r J_{0}\left(\phi_{j} r\right)+J_{1}\left(\phi_{j} r\right)\right) \\
+\frac{2 \mu C_{3}^{(j)}}{r \phi_{j}}\left(\left(\frac{\lambda+3 \mu}{\lambda+\mu} \cosh \left(\phi_{j} z\right)+\phi_{j} z \sinh \left(\phi_{j} z\right)\right) J_{1}\left(\phi_{j} r\right)\right. \\
\left.-\left(\phi_{j} r \frac{2 \lambda+3 \mu}{\lambda+\mu} \cosh \left(\phi_{j} z\right)+\phi_{j}^{2} r z \sinh \left(\phi_{j} z\right)\right) J_{0}\left(\phi_{j} r\right)\right) \\
+\frac{2 \mu C_{4}^{(j)}}{r \phi_{j}}\left(\left(\frac{\lambda+3 \mu}{\lambda+\mu} \sinh \left(\phi_{j} z\right)+\phi_{j} z \cosh \left(\phi_{j} z\right)\right) J_{1}\left(\phi_{j} r\right)\right. \\
\left.-\left(\phi_{j} r \frac{2 \lambda+3 \mu}{\lambda+\mu} \sinh \left(\phi_{j} z\right)+\phi_{j}^{2} r z \cosh \left(\phi_{j} z\right)\right) J_{0}\left(\phi_{j} r\right)\right),
\end{gathered}
$$

$$
\begin{array}{r}
\sigma_{\vartheta \vartheta}^{(j)}=-\frac{2 \mu}{r}\left(C_{1}^{(j)} \sinh \left(\phi_{j} z\right) J_{1}\left(\phi_{j} r\right)+C_{2}^{(j)} \cosh \left(\phi_{j} z\right) J_{0}\left(\phi_{j} r\right)\right) \\
-\frac{2 \mu C_{3}^{(j)}}{r \phi_{j}}\left(\left(\frac{\lambda+3 \mu}{\lambda+\mu} \cosh \left(\phi_{j} z\right)+\phi_{j} z \sinh \left(\phi_{j} z\right)\right) J_{1}\left(\phi_{j} r\right)\right. \\
\left.+\phi_{j} r \frac{\lambda}{\lambda+\mu} \cosh \left(\phi_{j} z\right) J_{0}\left(\phi_{j} r\right)\right) \\
-\frac{2 \mu C_{4}^{(j)}}{r \phi_{j}}\left(\left(\frac{\lambda+3 \mu}{\lambda+\mu} \sinh \left(\phi_{j} z\right)+\phi_{j} z \cosh \left(\phi_{j} z\right)\right) J_{1}\left(\phi_{j} r\right)\right. \\
\left.+\phi_{j} r \frac{\lambda}{\lambda+\mu} \sinh \left(\phi_{j} z\right) J_{0}\left(\phi_{j} r\right)\right) .
\end{array}
$$

The explicit expressions for the case in which the film and the substrate are both elastic are obtained in an analogous way.

\section{Numerical results}

In this section we present two numerical examples useful to analyze the behavior of the contact law and the stress distribution in terms of the film thickness and the elastic properties of the substrate and the film.

Consider therefore a thin isotropic film $(E=2 \mathrm{MPa}$ and $v=0.25)$ coated on a rigid substrate and in contact with a rigid sphere of radius $R=10 \mu \mathrm{m}$. In Figure 1 we show the contact law, for different thickness of the film $(h=5 \mu \mathrm{m}$ and $10 \mu \mathrm{m})$, 
obtained by using the solutions presented in Section 4 with Hertzian pressure distributions.

We remark that, when the thickness of the film is very large with respect to the contact area radius $a$ (i.e. $h \gg a$ ), we obtain numerical results in agreement with the theory of an elastic half-space with the properties of the film. On the other hand, when the thickness of the film is within the range between $a$ and $10 a$, the elastic response of the film changes for the presence of the rigid substrate [Yu et al. 1990; $\mathrm{Li}$ and Chou 1997]. Moreover, when the contact area radius is very large with respect to the film thickness (i.e. $h \ll a$ ), or in the case of a very soft material, the pressure distribution on the free surface of the film, and therefore the terms $A_{j}$ of the expansion (10), must be assigned by using models suitable to take into account the adhesion forces or the different pressure distribution [Maugis 1999].

Figure 2 shows the radial displacement in two ideal interface cases when the film thickness is $h=5 \mu \mathrm{m}$. The radial displacement, near the edge of the contact area, has a change of sign in the thickness; in particular, for $r=a$ and $z=0$ the radial displacement is negative (for both the interface cases) while it is positive in the interface for the frictionless case and zero for the perfectly bonded case.

Figures 3 and 4 show the normal, radial and tangential stress components for $P=2 \mu \mathrm{N}$; the maximum value of the normal compression stress is present on the

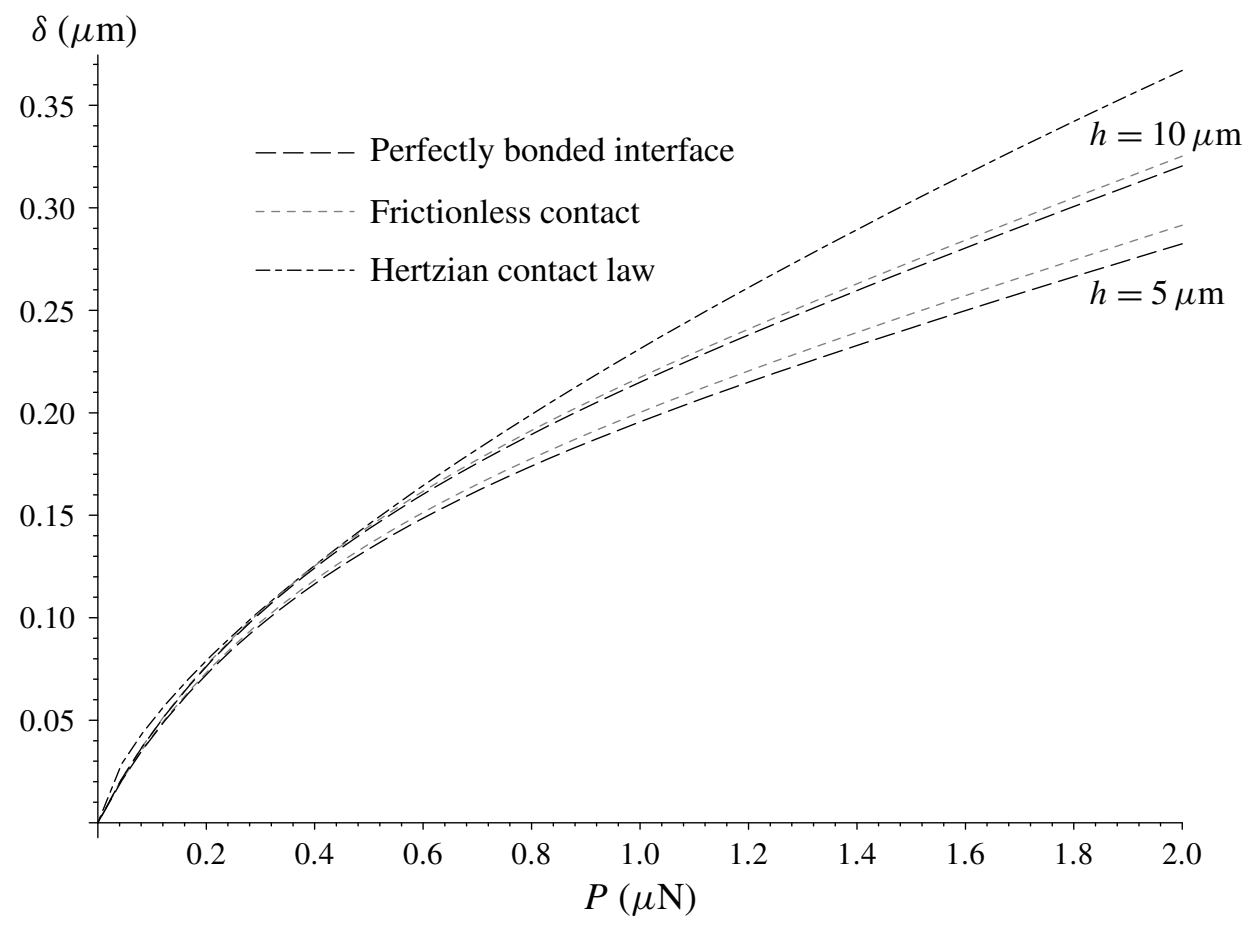

Figure 1. Contact laws for different film thicknesses. 
$u_{r}(\mathrm{~nm})$

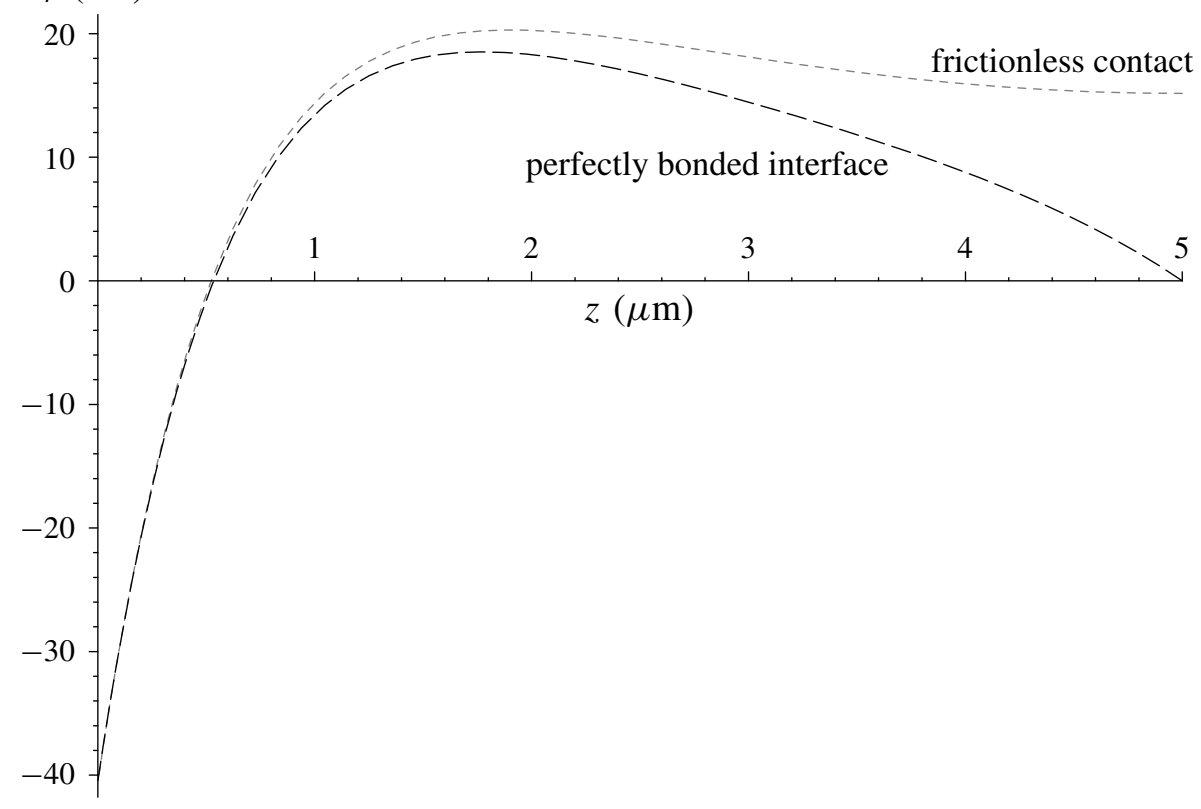

Figure 2. Radial displacement along the $z$-axis $(P=2 \mu \mathrm{N}, r=a)$.

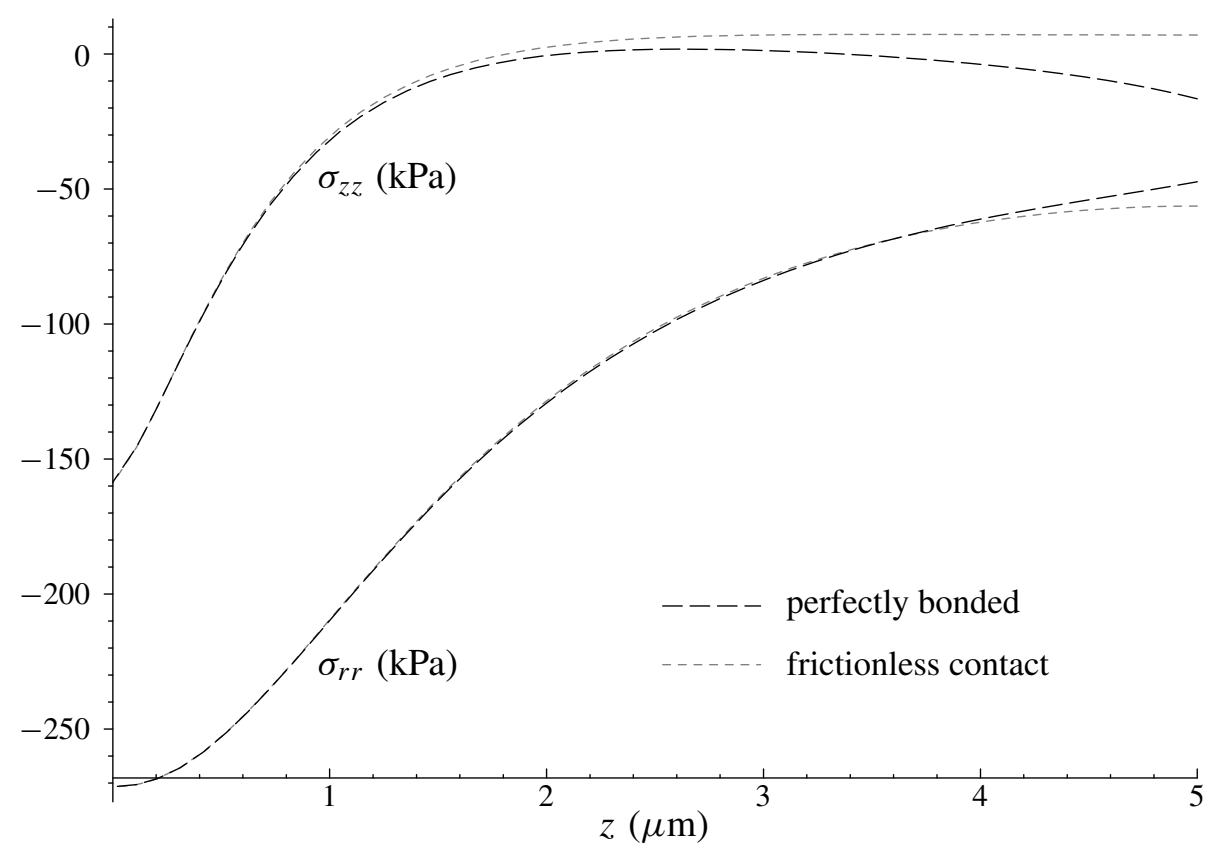

Figure 3. Radial and normal stress along the $z$-axis $(P=2 \mu \mathrm{N}$, $r=0)$. 


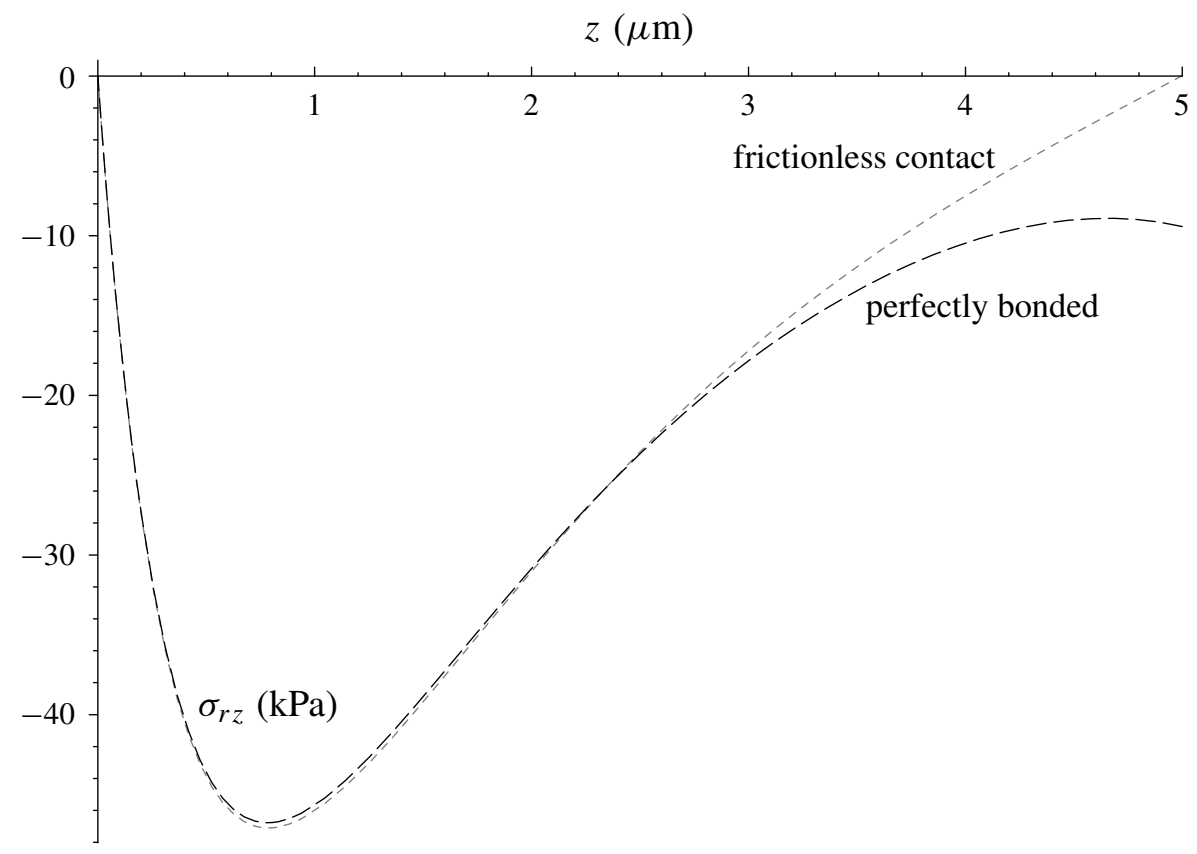

Figure 4. Shear stress along the $z$-axis $(P=2 \mu \mathrm{N}, r=a)$.

free surface and it is equal, for $r=0$, to the value of load pressure distribution $p_{o}$ (see Section 3.2.2). An analysis for different film thickness values shows that the component stress $\sigma_{z z}$ in the interface zone increases as the thickness of the film decreases.

The second example deals with different films on an elastic substrate. We have considered thin films (oxide $\mathrm{SiO}_{2}$ and tungsten W) coated on a silicon substrate ( $E=165 \mathrm{GPa}$ and $v=0.27$ ) and we have compared the numerical analytical results with the experimental data obtained in Chudoba et al. [2000].

Figure 5 shows the results for the oxide film $(E=72 \mathrm{GPa}$ and $v=0.17)$ with different thicknesses; the analytical model with Hertzian pressure distributions is suitable to describe the load-displacement behavior for elastic indentations with a spherical indenter for small values of the contact area radius $a$. In a first range, the contact law is in agreement with the elastic homogeneous half-space solution for oxide bulk material; in a second range, the contact law is different from the Hertzian contact law due to the substrate effects. Finally, for large contact area radius, the analytical results underestimate the experimental results to the order of $20 \%$; the reason for this fact is that the present analysis is limited to the idealized static problem in which other effects encountered in indentation problems are neglected (the different pressure distribution taking into account the substrate, adhesion forces, large deformation, and dynamic effects). In this case the Hertzian 


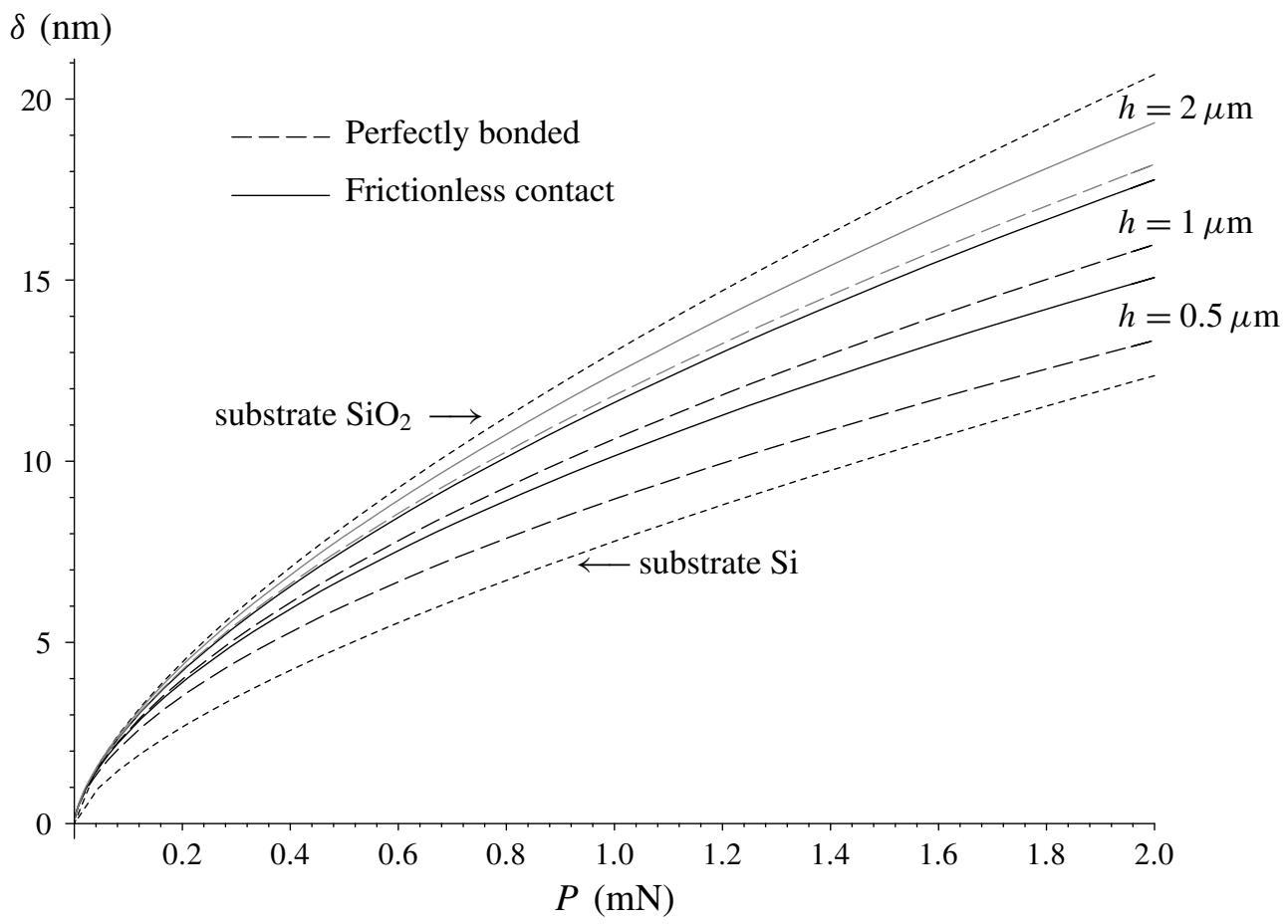

Figure 5. Contact law for $\mathrm{SiO}_{2}$ films on $\mathrm{Si}$ for different interface conditions.

contact law for a half-space with the film constitutive properties overestimate the experimental results.

The results for a tungsten film $(E=409 \mathrm{GPa}$ and $v=0.28)$ are shown in Figure 6 ; the analytical model with a Hertzian pressure is suitable to describe the loaddisplacement behaviour for small values of the contact area radius; for large values of $a$, the results obtained overestimate the experimental results since the Hertzian pressure distribution of the half-space is inaccurate to simulate the action of the indenter on the film for the soft substrate effect and a more appropriate pressure distribution needs to be assigned [Yang 2003; Chadwick 2002]. In this case the Hertzian contact law underestimates the experimental results.

More explicitly, the analytical results obtained for large $a$ show that the Hertzian overestimation for soft films on hard substrates and the underestimation for hard films on soft substrates are significant when the indentation depth is less than onetenth of the film thickness. This behaviour of over and underestimation by the Hertzian theory for soft and rigid films is in agreement with the results recently obtained by Wang et al. [2004], where a finite element analysis devoted to finding substrate effects at the nanoscale is presented.

Finally we remark that, for the cases discussed in this paper, we have numerically checked that, for value of $b$ greater than $10 a$, the values obtained become 


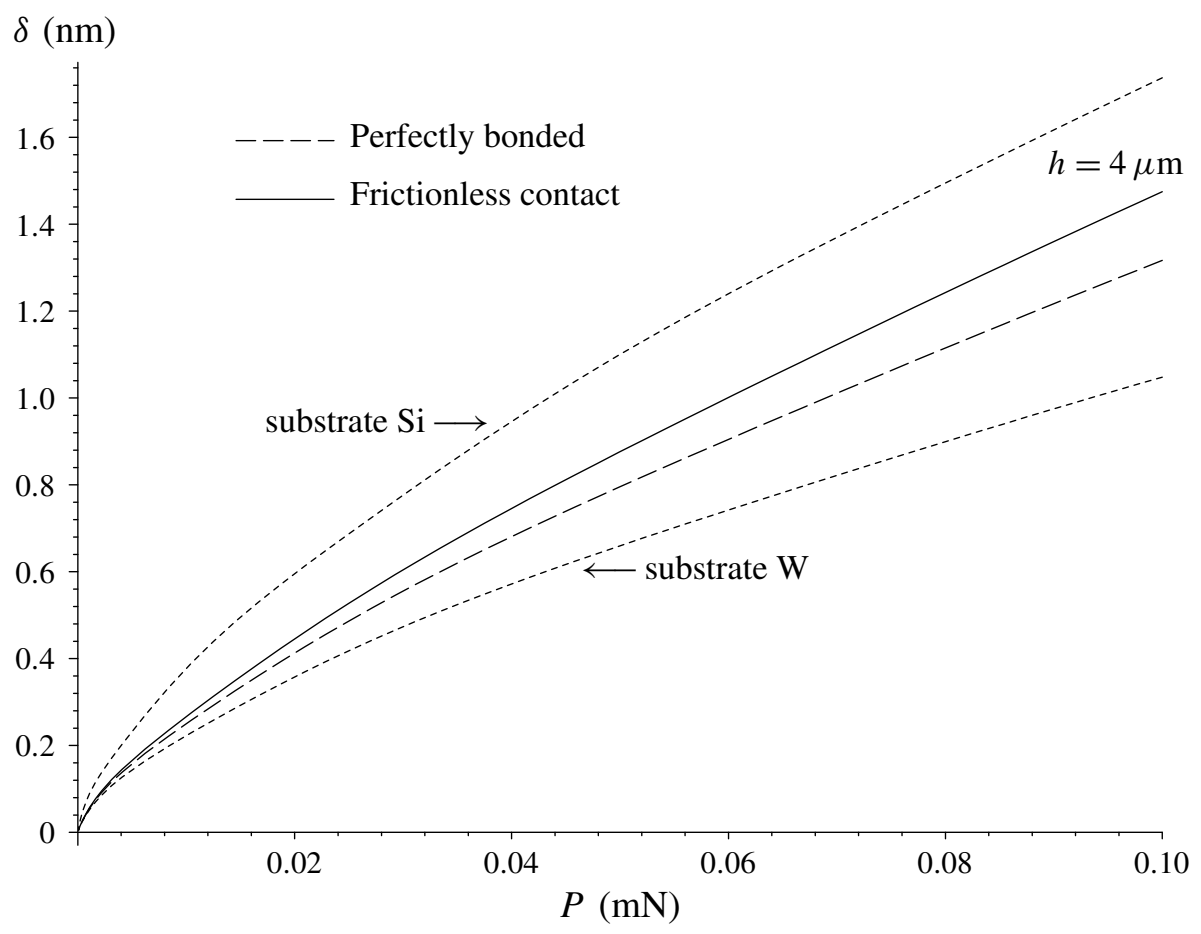

Figure 6. Contact law for tungsten film (W) on Si for different interface conditions.

numerically stable (by further increasing $b$ ) and so the specific choice assumed for $b$ becomes irrelevant. On the other hand, assuming a large $b$ requires considering Fourier-Bessel and Dini expansions up to very high terms, whose evaluation is unfruitful and very time-consuming. In our examples we assumed $b=10 a$ and we truncated the series when the data stabilized: typically with about 300 terms.

\section{Conclusions}

An elastic analysis has been performed to determine the displacement and the stress fields of thin-film coating/substrate system subjected to an axisymmetrical contact loading. The contact law presented in Section 4 approaches the half-space one when the film thickness is sufficiently large with respect to the contact area radius. If the film thickness is not large compared to the contact radius area, the response of the film is different because of the influence of the substrate. On the other hand, if the film thickness is very small compared to the contact radius, the classical pressure distribution in the half-space simulates inaccurately the action of the indenter on the film and a more appropriate pressure distribution has to be preassigned. Not only the classical pressure distributions under the indenter are 
potentially inaccurate, but so are the classical relations between contact area and load.

The solutions presented in Section 3, for different indenter shapes, furnish good suggestions for the study of the effect of adhesive forces that become more relevant when the coating bodies are small or compliant in nature. Finally, we remark that it is possible to extend the analysis of [Johnson and Sridhar 2001] adhesion theory for a thin elastic film on an elastic substrate by using the solution obtained in Section 4 [Maugis 1999].

\section{Acknowledgements}

The author thanks the reviewers for their contributions and particularly for the FE analysis which has given further support to the results presented in the paper.

This work was supported by MIUR Cofin Prin 2003: Interfacial damage failure in structural systems: applications to civil engineering and emerging research fields; and Cofin Prin 2005: Interfacial resistance and failure in materials and structural systems.

\section{References}

[Chadwick 2002] R. S. Chadwick, "Axisymmetric indentation of a thin incompressible elastic layer", SIAM J. Appl. Math. 62:5 (2002), 1520-1530. MR 2003d:74067

[Chudoba et al. 2000] T. Chudoba, N. Schwarzer, and F. Richter, "Determination of elastic properties of thin films by indentation measurements with a spherical indenter", Surf. Coat. Technol. 127:1 (2000), 9-17.

[Field and Swain 1993] J. S. Field and M. V. Swain, "A simple predictive model for spherical indentation", J. Mater. Res. 8:2 (1993), 297-306.

[Fischer-Cripps 2004] A. C. Fischer-Cripps, Nanoindentation, Springer, New York, 2004.

[Johnson and Sridhar 2001] K. L. Johnson and I. Sridhar, "Adhesion between a spherical indenter and an elastic solid with a compliant elastic coating", J. Phys. D, Appl. Phys. 34 (2001), 683-689.

[Li and Chou 1997] J. Li and T. W. Chou, "Elastic field of a thin-film/substrate system under an axysimmetric loading”, Int. J. Solids Struct. 34:35-36 (1997), 4463-4478.

[Matthewson 1981] M. J. Matthewson, "Axi-symmetric contact on thin compliant coatings", J. Mech. Phys. Solids 29:2 (1981), 89-113.

[Maugis 1999] D. Maugis, Contact, adhesion and rupture of elastic solids, Springer, New York, 1999.

[Oliver and Pharr 1992] W. C. Oliver and G. M. Pharr, "An improved technique for determining hardness and elastic modulus using load and displacement sensing indentation experiments", $J$. Mater. Res. 7:6 (1992), 1564-1583.

[Sneddon 1966] I. N. Sneddon, Mixed boundary value problems in potential theory, North-Holland, Amsterdam, 1966. MR 35 \#6853

[VanLandingham 2003] M. R. VanLandingham, "Review of instrumented indentation", J. Res. Natl. Inst. Stand. Technol. 108:4 (2003), 249-265. 
[Wang et al. 2004] M. Wang, K. M. Liechti, J. M. White, and R. M. Winter, "Nanoindentation of polymeric thin films with an interfacial force microscope", J. Mech. Phys. Solids 52:10 (2004), 2329-2354.

[Watson 1944] G. N. Watson, A treatise on the theory of Bessel functions, Cambridge University Press, Cambridge, 1944. MR 6,64a

[Yang 2003] F. Yang, "Axisymmetric indentation of an incompressible elastic thin film", J. Phys. D, Appl. Phys. 36:1 (2003), 50-55.

[Yu et al. 1990] H. Y. Yu, S. C. Sanday, and B. B. Rath, "The effect of substrate on the elastic properties of films determined by the indentation test-axisymmetric Boussinesq problem", J. Mech. Phys. Solids 38:6 (1990), 745-764.

Received 8 Dec 2005.

ROBERTA SBURLATI: sburlati@diseg.unige.it

Dipartimento di Ingegneria Strutturale e Geotecnica, Facoltà di Ingegneria, Università di Genova, Via Montallegro 1, I-16145 Genova, Italy

http://www.diseg.unige.it/staff/sburlati/rsburlati 